\title{
Curative effect of stereotactic body radiotherapy for unresectable massive primary liver cancer
}

\author{
YA-ZHENG DANG ${ }^{*}$, XIN LI ${ }^{*}$, SHI-GAO HUANG ${ }^{*}$, WAN-LING LU, \\ GUODONG WANG, DONGXIAN ZHANG and QIAN-YUN WANG
}

\begin{abstract}
Department of Radiation Oncology, 323 Hospital of People's Liberation Army, Xi'an, Shaanxi 710054, P.R. China
\end{abstract}
Received May 28, 2016; Accepted February 28, 2017

DOI: $10.3892 / \mathrm{mco} .2017 .1255$

\begin{abstract}
The aim of this study was to evaluate the curative effect of gamma knife stereotactic body radiotherapy (SBRT) for unresectable massive primary liver cancer. A total of 69 patients with unresectable massive $(>10 \mathrm{~cm})$ primary liver cancer who were treated by SBRT at the Department of Radiation Oncology of the 323 Hospital of People's Liberation Army (Xi'an, China) between October, 2006 and October, 2010, were analyzed. According to the Union for International Cancer Control TNM staging guidelines, the patients were graded as stage T1 $(n=8)$, T2 $(n=12), T 3(n=21)$ and T4 $(n=28)$. None of the patients had lymph node metastasis, whereas 45 patients had portal vein tumor thrombosis. The Child-Pugh class was A $(n=49), B(n=15)$ and C $(n=5)$. The visible tumor volume \pm standard deviation was $810 \pm 213 \mathrm{~cm}^{3}$. The patients received a total radiation dose of 50-60 Gy, with a dose fractionation of 4-6 Gy/fx, administered for a total of 9-12 times, 2-5 times/week. A total of 8 patients succumbed to the disease within 3 months after gamma knife treatment and were not included in the evaluation of the curative effect. The total effectiveness rate was 59.0\% (36/61) and the median survival was 17.4 months for all the patients included in the study. The 1-, 2-, and 3-year overall survival rates were 71, 30 and $22 \%$, respectively. In conclusion, SBRT appears to be effective for unresectable massive primary liver cancer.
\end{abstract}

\section{Introduction}

The clinical tumor size of middle-to-late-stage primary liver cancer is estimated by its maximum diameter. Hepatocellular

Correspondence to: Dr Shi-Gao Huang, Department of Radiation Oncology, 323 Hospital of People's Liberation Army, 6 Construction West Road, Xi'an, Shaanxi 710054, P.R. China

E-mail: huangshigao2010@aliyun.com

*Contributed equally

Key words: massive primary liver tumor, radiation therapy, gamma knife, stereotactic body radiotherapy carcinomas (HCCs) sized from $>5$ to $<10 \mathrm{~cm}$ are described as large, whereas those $\geq 10 \mathrm{~cm}$ as massive. Multimodal comprehensive treatment has been used for unresectable large live cancer, but without a distinct curative effect; the outcome of massive HCC treated with non-surgical methods is not satisfactory. Transcatheter arterial chemoembolization (TACE) is contraindicated in patients with HCC invasion of the first branch or main trunk of the portal vein, as it may cause extensive liver infarction, resulting in poor prognosis (1). Another limitation of TACE, as reported by Arata et al (2), is that local control of HCCs sized $\geq 5 \mathrm{~cm}$ is often difficult. These two limitations are responsible for negative survival data in initial randomized control trials of TACE (3). At present, radiation therapy (RT) is applied as palliative treatment for liver cancer, mainly as three-dimensional conformal RT (3D-CRT). 'Super gamma knife' technology is one of the most effective methods for solid tumors, has the advantages of high precision and high single therapeutic dose, and it may improve the effects of palliative care in terminal-stage liver cancer. In the present study, 69 cases of primary unresectable liver cancer who underwent gamma knife stereotactic body radiotherapy (SBRT) were retrospectively analyzed to investigate the prognostic factors and curative effect of this type of radiotherapy, providing the basis for further clinical applications.

\section{Patients and methods}

Patient characteristics. This study included 69 patients with primary liver cancer admitted to the 323 Hospital of People's Liberation Army (Xi'an, China) between October, 2006 and October, 2010. A total of 22 patients received TACE prior to gamma knife therapy. All the patients received RT doses of 50-60 Gy in daily fractions of 4-6 Gy. The eligibility criteria comprised the following: i) Unresectable HCC with portal vein tumor thrombosis (PVTT) in the first branch or the main trunk; ii) an HCC sized $\geq 10 \mathrm{~cm}$; iii) absence of extrahepatic metastasis (4) and no ascites or medical control of ascites; and iv) an Eastern Cooperative Oncology Group performance status of 0-2 (5). A total of 45 HCC patients with portal vein tumor thrombosis (PVTT) were initially diagnosed by liver biopsy histological examination and 24 patients were initially diagnosed on dynamic helical computed tomography (CT) using contrast medium or by magnetic resonance imaging (MRI) and measurement of $\alpha$-fetoprotein levels, then reconfirmed by CT 
during hepatic arteriography and CT during arterial portography in each case. Patients with diabetes were excluded. The patient characteristics are summarized in Table I. This study was approved by the Ethics Committee of the 323 Hospital of PLA and all the patients provided written informed consent prior to enrolment.

Treatment planning. RT was performed using the 'super gamma-knife' SGS-I Stereotactic Gamma-Ray system (Huiheng Medical Inc., Shenzhen, China), which focused on whole-body radiation, and the UNICORN 3D Treatment Planning System (Huiheng Medical Inc.) for the design. RT plans were reviewed using our department's treatment planning software (Huiheng Medical Inc.). Individual gross tumor volumes (GTVs) for each liver tumor and nodal lesion visualized on positron emission tomography were generated on planning CTs. Both simulation and treatment-planning CTs were performed during breath holding. The patients were instructed to breathe quietly, as RT was repeatedly delivered during breath holding at end-expiration for 10-15 sec at a time. Using these data, the whole liver, main tumor, PVTT, and other hepatic tumors were contoured for each patient with reference to the MRI or diagnostic enhanced CT images taken within a week prior to treatment planning (Fig. 1A). Outlining of the target area-GTV was performed by a medical physicist and the planning target volume (PTV) was extended $0.5 \mathrm{~cm}$ outside the GTV. The organs at risk included the normal liver, duodenal pancreas, kidney and spinal cord. It was ensured that the 50\% isodose curve covered the PTV and that the radiation delivered to normal tissue did not exceed the tolerance dose (Fig. 2). A dose-volume histogram (DVH) was drawn to evaluate the square and optimize the radiation scheme. The RT prescription dose was as follows: 4-6 Gy/fx, for a total of 9-12 times, 2-5 times/week, up to a total dose of 50-60 Gy. Organs of interest were avoided and the treatment plan optimization index was determined with DVH; 40 or $50 \%$ DVH surrounded the PTV (Fig. 3). The dose administered to adjacent target organs did not exceed 25 Gy (tolerance dose). During treatment, the patients were administered static drops of liquorice anhydride, while the use of chemicals was avoided.

Curative effect evaluation. Efficacy evaluation was performed according to the World Health Organization curative effect evaluation standards of solid tumors (4). Three months after RT, the patients were evaluated by abdominal MRI. After treatment completion, reexamination was performed every 3 months for 1 year and every 6 months thereafter. Reexamination included blood tests, liver and kidney function tests, serum electrolyte levels, abdominal MRI and abdominal ultrasound.

Statistical analysis. SPSS software, version 19 (SPSS Inc., Chicago, IL, USA) was used to perform the statistical analyses. The curative effect observation index was overall survival estimated with Kaplan-Meier curve analysis.

\section{Results}

Curative effect. A total of 8 patients succumbed to the disease within 3 months after gamma knife treatment and were not
Table I. Patient characteristics $(\mathrm{n}=69)$.

\begin{tabular}{lc}
\hline Characteristics & $\mathrm{N}(\%)$ \\
\hline Gender & \\
Male & $53(76.8)$ \\
Female & $16(23.2)$ \\
Age, years & \\
Median & 54 \\
Range & $28-89$ \\
T stage & \\
T1 & $8(11.6)$ \\
T2 & $12(17.4)$ \\
T3 & $21(30.4)$ \\
T4 & $28(40.6)$ \\
PVTT & $45(65.2)$ \\
Tumor size, cm & \\
Mean & $11.0(9.0-21.0)$ \\
Median & 10.1 \\
Child- Pugh class & \\
A & $49(71.0)$ \\
B & $15(21.7)$ \\
C & $5(7.3)$ \\
RT dose, Gy & \\
Median & 70.0 \\
Range & $69.4-70.6$ \\
\hline
\end{tabular}

PVTT, portal vein tumor thrombosis; RT, radiotherapy.

included in the evaluation of the curative effect. Among the remaining 61 patients, 8 had non-cancerous ascites, hepatomegaly, a 2 -fold increase in alkaline phosphatase levels and/or at least a 5-fold increase in transaminase levels, which it was diagnosed as radiation-induced liver disease (RILD), according to the diagnostic standards reported in the literature $(6,7)$. A total of 7 patients achieved a complete response (CR), 29 achieved a partial response (PR), 24 had stable disease (SD) and 9 developed progressive disease (PD), including 5 cases of local progression and 4 of intrahepatic metastasis. The total effectiveness rate $(\mathrm{CR}+\mathrm{PR})$ was $59.0 \%$ (36/61), the intrahepatic metastasis rate was $14.8 \%(9 / 61)$ and the incidence of RILD was $13.1 \%(8 / 61)$.

Survival analysis. The overall survival of patients with massive primary liver cancer following RT is shown in Fig. 4. The median survival was 17.4 months for all patients included in the study and the 1-, 2- and 3-year overall survival rates were 71,30 and $22 \%$, respectively.

Adverse reactions. The severity of the adverse reactions was scored for each patient using the National Cancer Institute (NCI) Common Toxicity Criteria (CTC), version 2.0 (6), the grading system of the Radiation Therapy Oncology Group (RTOG) (7), and the decrease in the white blood cell (WBC) and platelet (PLT) counts. Other widely used scoring systems include those of the RTOG and European Organization for 

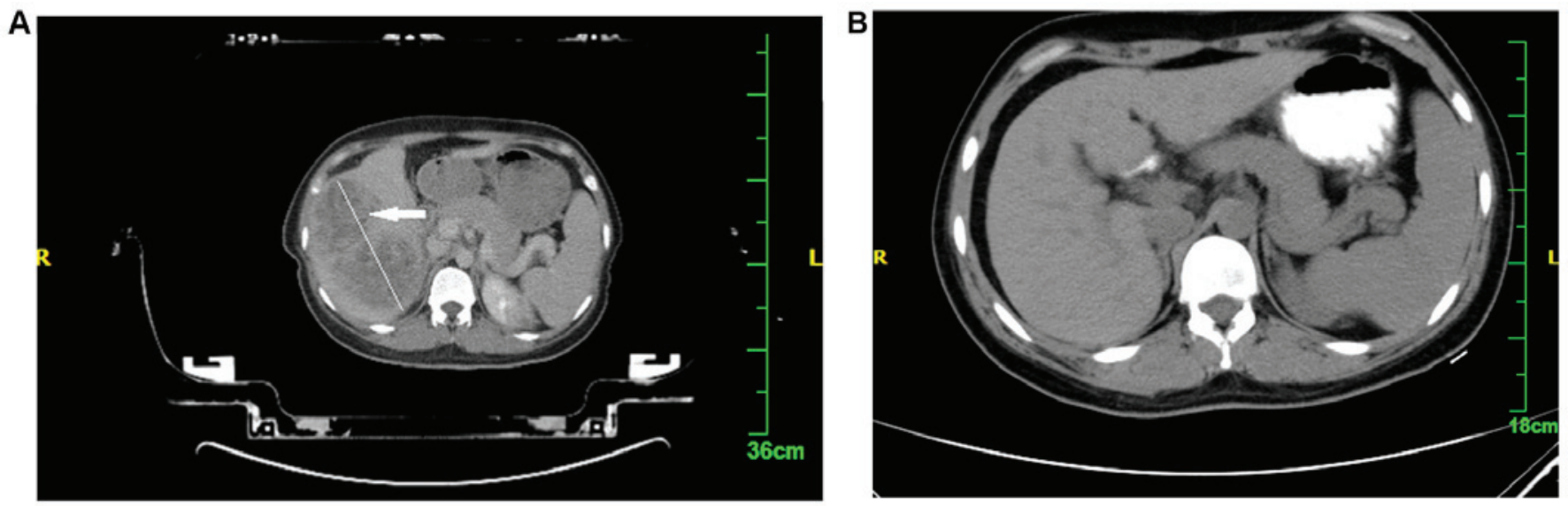

Figure 1. Computed tomography images of a 28 -year-old woman with hepatocellular carcinoma showing (A) a giant tumor (10x6 cm) in the liver (arrow) prior to radiotherapy and (B) disappearance of the tumor body, with no tumor metastasis oe residual tumor after radiation treatment.

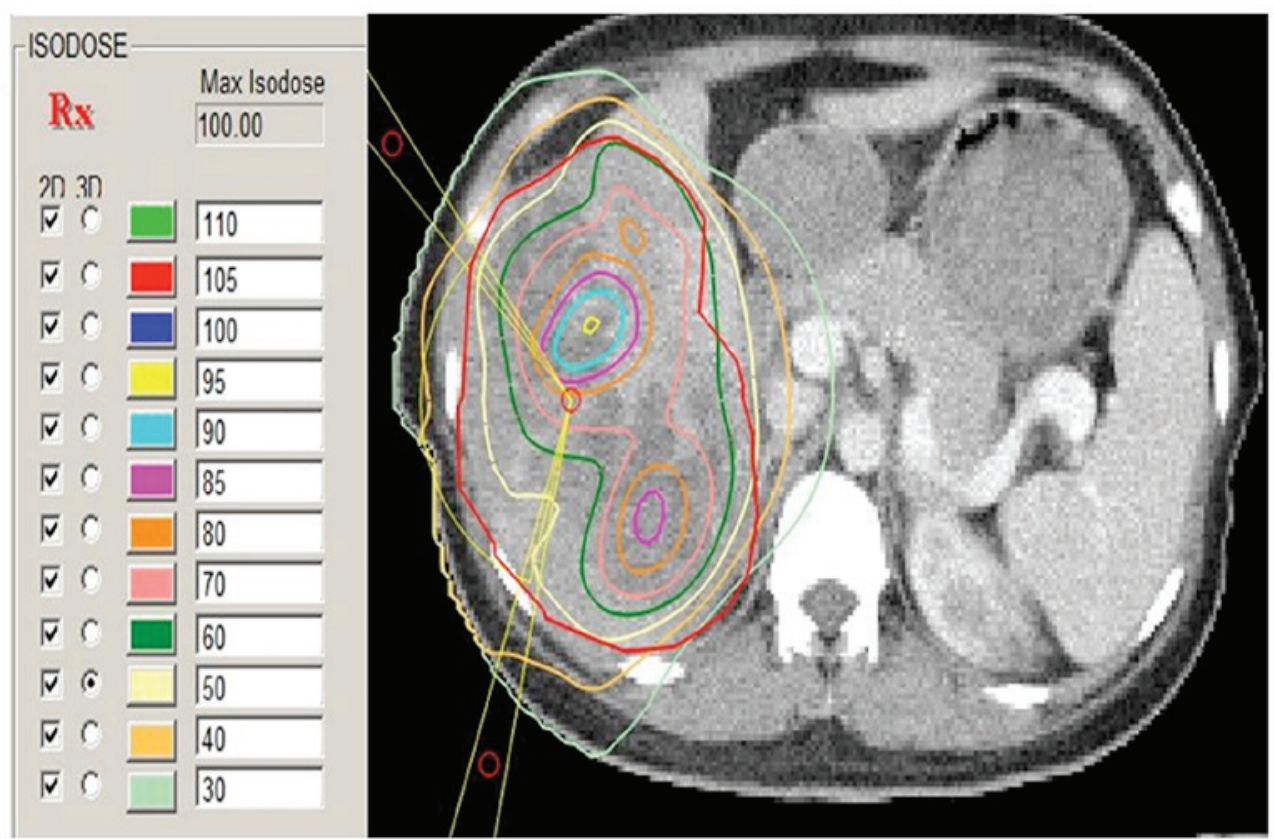

Figure 2. Images of a 28-year-old woman with hepatocellular carcinoma, 12-colour stand for various isodose lines of 2D chart of the planning target volume. The dose gradually increased from the peripheral circle to the center.

Research and Treatment of Cancer, and the Late Effects Normal Tissue Task Force (8). These scales include four separate elements, representing subjective, objective, management and analytical evaluation of injury (Table II).

As shown in Table III, 22 patients had acute liver adverse reactions classified according to NCI-CTC 2.0 as grade $1(n=5)$, grade $2(n=13)$ and grade $3(n=4)$. Treatment with compound glycyrrhizin injection was administered to these patients and the liver function returned to normal; however, in 8 cases the liver function did not recover and the patients succumbed to the disease 3 months after RT. A total of 57 patients exhibited digestive tract reactions classified by RTOG as grade $0(n=15)$, $1(n=23)$ and $2(n=19)$. A decrease in the WBC count was observed in 52 cases (grade 1 in 29 and grade 2 in 23 cases) and a decrease in the PLT count was observed in 35 cases (grade 1 in 24, grade 2 in 8 and grade 3 in 3 cases).

\section{Discussion}

Historically, the liver has been considered to be a relatively radiosensitive organ and it may be difficult to achieve the radiation doses required to eradicate gross tumors without causing RILD, which generally develops $\sim 4$ weeks following RT (9). The higher incidence of RILD in this study may be associated with the sizeable tumor and the single integral high dose and high total dose of RT. The curative effect of massive liver cancer was significant. As seen in Fig. 1, showing a pre- and post-treatment CT, the liver displayed a sizeable shadow prior to treatment, whereas the shadow completely disappeared following RT. The reason for this significant shrinking or disappearance of the tumor may be associated with high division of a single dose and the total dose. 

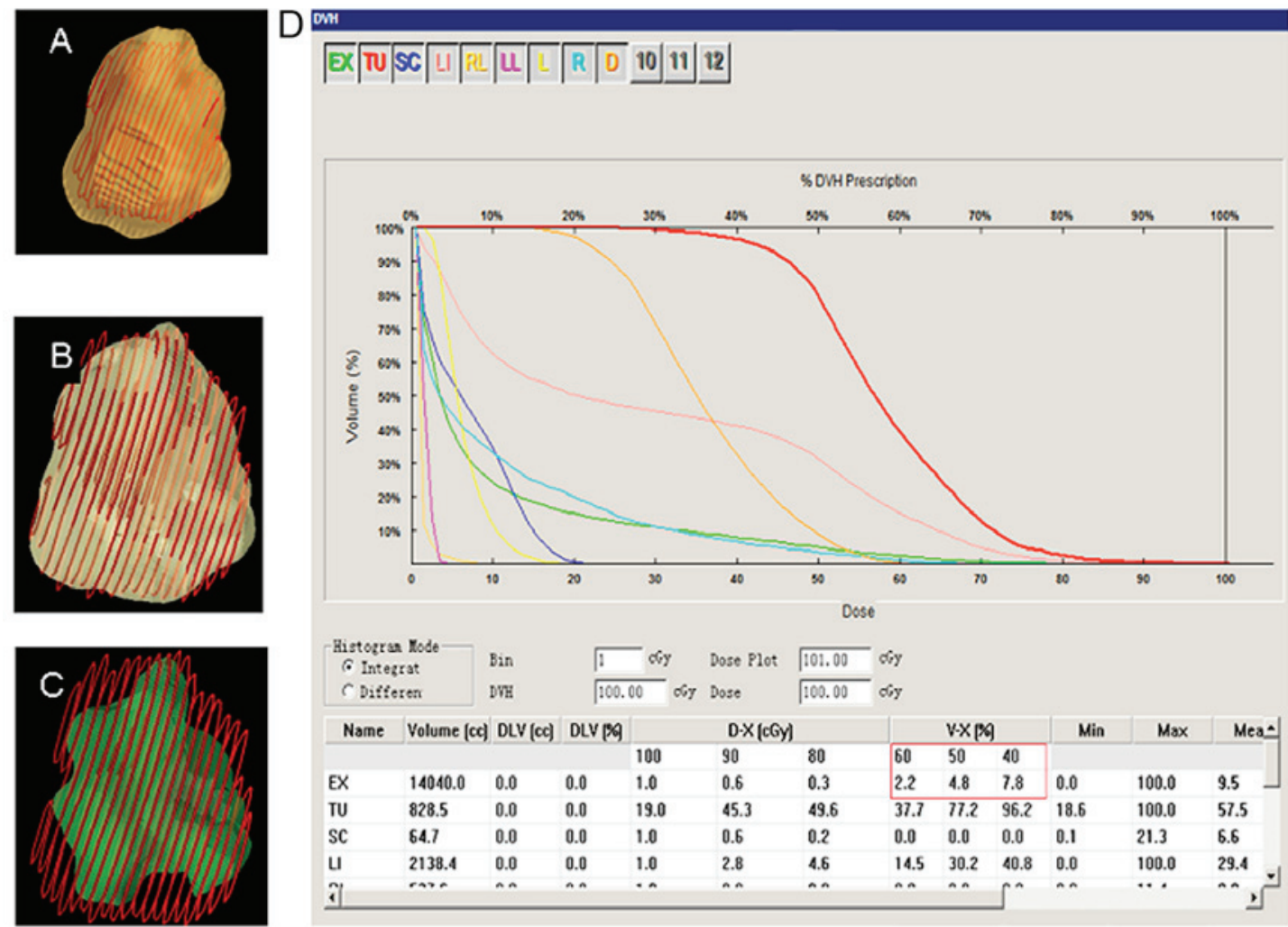

Figure 3. Images of a 28-year-old woman with hepatocellular carcinoma. Three-dimensional chart of the (A) $40 \%$, (B) 50\% and (C) $60 \%$ isodose covering the planning target volume (PTV). (D) Isodose curve showing that 40, 50 and $60 \%$ of the isodose covered $96.2,77.2$ and $37.7 \%$ of the PTV, respectively (red box).

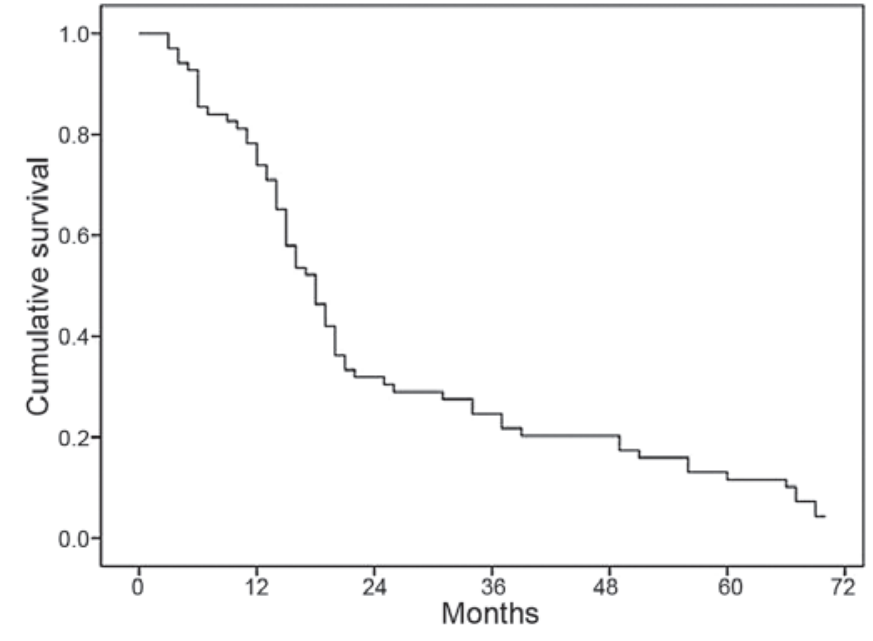

Figure 4. Kaplan-Meier analysis of overall survival following stereotactic body radiotherapy for hepatocellular carcinoma.

Child-Pugh classification, which was first introduced by the Child in 1964 (10), is commonly used to quantitatively assess liver reserve function in patients with cirrhosis. This classification divides patients in different groups based on five indicators (general status, ascites, serum bilirubin, serum albumin and prothrombin time) scored with 1,2 and 3 points. The score of the five indicators is added (the lowest score is 5 points and the highest score is 15 points) and liver reserve function is classed as A, B or C, indicating three different levels of severity of liver damage (the higher the score, the worse the liver reserve function). Patient prognosis is considered to be associated with liver function according to the Child-Pugh classification, with patients classed as Child-Pugh A having a good prognosis.

Intermediate- or advanced-staged liver cancer patients may have cirrhosis and poor liver function, and the majority of patients who are not considered to be candidates for surgical treatment may receive comprehensive treatment. TACE has been considered as the standard optimal treatment for unresectable primary liver cancer (11), but its curative effect is affected by the patient's general condition, tumor progression and blood supply of tumor tissue. As residual tumor cells remain in the majority of the lesions, the long-term outcome is not satisfactory (12). In recent years, TACE combined with conventional RT have achieved a good curative effect. Seong et al (13) reported 30 cases treated with TACE followed by conventional RT (normalized total dose, $44 \mathrm{~Gy}$ ), and the reported results were a median survival of 17 months and a 3 -year survival rate of $22.2 \%$. In the present study, 69 patients with massive liver cancer $(\geq 10 \mathrm{~cm})$, advanced TNM stage, poor liver function and PVTT (45/61), were unable to receive conventional TACE treatment. In such cases, RT is considered the best palliative treatment option, but the patients' prognosis is poorer. Xiong et al (14) reported on 69 cases of massive HCC treated with 3D-CRT (fraction dose 4-8 Gy, for 7-15 times, to a total dose of 53.6 $\pm 6.6 \mathrm{~Gy}$ ). The 1-, 2- and 3-year survival rates were 41, 20 and 17\%, respectively, but there was no analysis of the curative effect of RT combined with TACE. Therefore, the curative effect of TACE for massive liver cancer is not satisfactory, and data on 
Table II. Criteria for grading by the toxicity scoring system.

\begin{tabular}{|c|c|c|c|c|}
\hline Scoring system & 0 & 1 & 2 & 3 \\
\hline NCI-CTC 2.0 & - & $\begin{array}{c}\text { 1.26- } 2.5 \text { times the upper limit } \\
\text { of normal ALT value }\end{array}$ & $\begin{array}{l}\text { 2.6-5 times the upper } \\
\text { limit of normal ALT value }\end{array}$ & $\begin{array}{l}\text { 5-10 times the upper } \\
\text { limit of normal ALT value }\end{array}$ \\
\hline RTOG & $\begin{array}{c}\text { No nausea } \\
\text { and vomiting }\end{array}$ & Nausea & Vomiting can be controlled & - \\
\hline LENT/SOMA & - & - & - & - \\
\hline $\begin{array}{l}\text { WBC decline in } \\
\text { grading }\left(\times 10^{9}\right)\end{array}$ & - & $\begin{array}{c}\text { WBC at } \\
(3-3.9) \times 10^{9}\end{array}$ & $\begin{array}{c}\text { WBC at } \\
(2-2.9) \times 10^{9}\end{array}$ & - \\
\hline $\begin{array}{l}\text { Platelet decline in } \\
\text { grading }\left(\times 10^{9}\right)\end{array}$ & - & 75-99 & $50-74$ & $28-49$ \\
\hline
\end{tabular}

NCI-CTC, National cancer institute common toxicity criteria; ALT, alanine transaminase; RTOG, radiation therapy oncology group; LENT/SOMA, late effects normal tissue task force/subjective, objective, management, and analytic scales; WBC, white blood cell.

Table III. Demographic and clinical characteristics of the patients included in data analysis.

\begin{tabular}{lrrrr}
\hline & \multicolumn{4}{c}{ Scoring rate $(\mathrm{n})$} \\
\cline { 2 - 5 } Variables & \multicolumn{1}{c}{0} & 1 & 2 & 3 \\
\hline NCI-CTC 2.0 (n) & - & 5 & 13 & 4 \\
RTOG & 15 & 23 & 19 & - \\
WBC decline in grading $\left(\times 10^{9}\right)$ & - & 29 & 23 & - \\
Platelet decline in grading $\left(\times 10^{9}\right)$ & - & 24 & 8 & 3 \\
\hline
\end{tabular}

NCI-CTC, national cancer institute common toxicity criteria; RTOG, radiation therapy oncology group; WBC, white blood cell.

the curative effect of TACE combined with RT have not yet been reported.

Super gamma knife SBRT is stereotactic radiosurgery combined with 3D-CRT technology. Compared with traditional body gamma knife accurate positioning, SBRT uses concentric dose control rings at different distances from the PTV, with the dose increasing from the outer to the inner ring. 3D-CRT may be used for larger tumors, which is advantageous in terms of increasing the target dose and protecting normal tissues. Due to the characteristics of the focus and the high dose, SBRT significantly improves the curative effect and reduces the incidence of the radiation reactions and the extent of normal tissue damage. Dang et al (15) reported the curative effect of SBRT on hepatic hilar carcinoma and confirmed that the technology of gamma knife treatment of primary liver cancer is a safe and reliable treatment, effective in improving patient survival. Normalized total dose (NTD) was defined as the biologically equivalent total dose, normalized to $2 \mathrm{~Gy}$ per fraction. The association of biologically effective dose (BED) with NTD is illustrated as NTD $=\mathrm{BED} /[1+\mathrm{d} /(\alpha / \beta)](16)$. According to Fletcher (17), if $\alpha / \beta=10 \mathrm{~Gy}$, conventional single dose $(\mathrm{d})=2 \mathrm{~Gy}$, the GTV dose should be as follows: When the NTD for small lesions was $60 \mathrm{~Gy}$, the BED was $72 \mathrm{~Gy}$, and when the NTD for tumors sized $\geq 10 \mathrm{~cm}$ was $50-60 \mathrm{~Gy}$, the BED was 60-72 Gy, as the dose gradually increased from the periphery to the center (Fig. 2). Although the NTD for tumors sized $\geq 10 \mathrm{~cm}$ is lower compared with the NTD for small lesions, the opposite stands for the dose to the central area of the tumor, as the single irradiation dose for tumors sized $\geq 10 \mathrm{~cm}$ is higher compared with that for small lesions.

In this study, the dose to the periphery of the tumor was 50-70 Gy, which was equivalent to an NTD of $65 \mathrm{~Gy}$, but the dose to the central area of the tumor may reach $>90$ Gy. The therapeutic dose was lower than the conventional radiation dose. The total effectiveness rate $(\mathrm{CR}+\mathrm{PR})$ was $51 \%$ and the 1-, 2- and 3-year overall survival rates were 45, 24 and 19\%, respectively. The median survival was 13 months, which was superior to that with 3D-CRT, due to the high central dose. However, there was an increased incidence of adverse reactions, such as acute liver toxicity and gastrointestinal reactions (27 and 73\%, respectively), a 63\% decrease in the WBC count and a $41 \%$ decrease in the PLT count.

Although gamma knife treatment for massive HCC appears to be effective, its curative effect is not satisfactory. As the majority of the patients are at an advanced stage, with compromised liver function and poor general status, only few patients may be suitable candidates for gamma knife treatment. Further research for methods to improve the curative effect of treatment is required.

\section{References}

1. Yamada R, Sato M, Kawabata M, Nakatsuka H, Nakamura K and Takashima S: Hepatic artery embolization in 120 patients with unresectable hepatoma. Radiology 148: 397-401, 1983.

2. Arata S, Tanaka K, Okazaki H, Kondo M, Morimoto M, Saito S, Numata K, Nakamura S and Sekihara H: Risk factors for recurrence of large HCC in patients treated by combined TAE and PEI. Hepatogastroenterology 48: 480-485, 2001.

3. A comparison of lipiodol chemoembolization and conservative treatment for unresectable hepatocellular carcinoma. Groupe d'Etude et de Traitement du Carcinome Hépatocellulaire. N Engl J Med 332: 1256-1261, 1995.

4. WHO: Handbook for reporting the results of cancer treatment. Offset Publication No. 48. World Health Organization. Geneva, pp34-35, 1979.

5. Oken MM, Creech RH, Tormey DC, Horton J, Davis TE, McFadden ET and Carbone PP: Toxicity and response criteria of the Eastern Cooperative Oncology Group. Am J Clin Onco 12: 649-655, 198. 
6. Cancer Therapy Evaluation Program. Available at http://ctep .cancer.gov/reporting/ctc.html. Accessed February 25, 2010.

7. Cox JD, Stetz J and Pajak TF: Toxicity criteria of the radiation therapy oncology group (RTOG) and the European organization for research and treatment of cancer (EORTC). Int J Radiat Oncol Biol Phys 31: 1341-1346, 1995.

8. Pavy JJ, Denekamp J, Letschert J, Littbrand B, Mornex F, Bernier J, Gonzales-Gonzales D, Horiot JC, Bolla M and Bartelink H: EORTC Late Effects Working Group. Late effects toxicity scoring: The SOMA scale. Int J Radiat Oncol Biol Phys 31: 1043-1047, 1995.

9. Lawrence TS, Robertson JM, Anscher MS, Jirtle RL, Ensminger WD and Fajardo LF: Hepatic toxicity resulting from cancer treatment. Int J Radiat Oncol Biol Phys 31: 1237-1248, 1995.

10. Shetty K, Rybicki L and Carey WD: The Child-Pugh classification as a prognostic indicator for survival in primary sclerosing cholangitis. Hepatology 5: 1049-1053, 1997.

11. Glouse ME, Stokes KR, Kruskal JB, Perry LJ, Stuart KE and Nasser IA: Chemoembolization for hepatocellular carcinoma: Epinephrine followed by a doxorubicin-ethiodized oil emulsion and gelatin sponge powder. J Vasc Interv Radiol 4: 717-725, 1993
12. Nakai M, Sato M, Kawai N, Minamiguchi H, Masuda M, Tanihata H, Takeuchi T, Terada M and Kishi K: Hepatocellular carcinoma: Involvement of the internal mammary artery. Radiology 219: 147-152, 2001.

13. Seong J, Park HC, Han KH, Lee DY, Lee JT, Chon CY, Moon YM and Suh CO: Local radiotherapy for unresectable hepatocellular carcinom a patients who failed with transcatheter arterial chemoembolization. Int J Radia Oncol Biol Phys 47: 1331-1335, 2000.

14. Xiong LS, Dong ZX, Liang JG, Long C and Li YY: Three-dimensional conformal radiotherapy(3-DCRT) for 69 cases of massive primary liver cancer. Chin Oncol 16: 478-479, 2006.

15. Dang YZ, Huang SG, Lu WL, Wu FW and Wang QY: Curative effect of stereotactic body radiotherapy on hepatic hilar carcinoma. Mol Clin Oncol 2: 1135-1138, 2014

16. Park C, Papiez L, Zhang S, Story $M$ and Timmerman RD: Universal survival curve and single fraction equivalent dose: Useful tool in understanding potency of ablative radiotherapy. Int J Radiat Oncol Biol Phys 70: 847-852, 2008.

17. Fletcher GH: Clinical dose-response curve of subclinical aggregates of epithelial cells. J Radiol Electrol Med Nucl 53: 201-206, 1972. 